\title{
ASSESSMENT OF PRESCRIBING PATTERN AMONG ORTHOPEDIC IN-PATIENTS USING WHO PRESCRIBING INDICATORS
}

\author{
ABHILASH S ${ }^{1}$, RASHMI R RAO ${ }^{2 *}$, SIVAGUHA YADUNATH P $^{1}$ \\ ${ }^{1}$ Undergraduate, Department of Pharmacology, Kasturba Medical College, Mangalore, Manipal Academy of Higher Education, Manipal - \\ 575 001, Karnataka, India. ${ }^{2}$ Assistant Professor, Department of Pharmacology, Kasturba Medical College, Mangalore, Manipal Academy of \\ Higher Education, Manipal - 575 001, Karnataka, India. Email id: rashmi.rao@manipal.edu
}

Received: 16 August 2018, Revised and Accepted: 25 September 2018

ABSTRACT

Objective: Evaluating the prescribing indicators regularly help to monitor the prescriptions to reduce indiscriminate use of drugs. The present study was conducted to explore the prescribing pattern in orthopedic in-patients using the WHO prescribing indicators in a tertiary care teaching hospital, Mangalore.

Methods: Data was collected from case records of orthopedic in-patients regarding patient demographics, diagnosis, and details of drugs in a structured pro forma and analyzed for the WHO prescribing indicators using descriptive statistics. Values are expressed as mean \pm standard deviation, percentages as applicable.

Results: A total of 379 patient prescriptions were included in the study which consisted of 2195 drugs. Of the 379 patients, 221 (58.3\%) were male and $158(41.7 \%)$ were female. The mean age group of the patients was 44 years. Acute trauma such as fractures, tendon damage, and disc prolapse $(57.8 \%)$ were the most common indications for admission. Analgesics were the most commonly prescribed group of drugs comprising $27.28 \%$ of the prescriptions followed by multivitamins (20.68\%) and proton-pump inhibitors (17.12\%). The most commonly prescribed analgesic was nonsteroidal antiinflammatory drugs (86.81\%). Most of the drugs (65.80\%) were administered through the oral route and $40.31 \%$ of drugs were fixed-dose combinations. The mean number of drugs per prescription was $5.79 \pm 2.59$. Antibiotics were prescribed in $60.4 \%$ of the prescriptions. $76.3 \%$ of prescriptions were with injectables. Use of drugs by generic name was $7.7 \%$ and only $44 \%$ of drugs were from the national list of essential medicines 2015 .

Conclusion: The study provides an insight into the prescribing pattern in orthopedic in-patients. It highlights the importance of emphasizing rational drug prescribing and toward improving awareness of the physicians and medical students to the WHO recommended standards on prescribing indicators.

Keywords: Prescribing pattern, WHO prescribing indicators, Orthopedic in-patients.

(C) 2018 The Authors. Published by Innovare Academic Sciences Pvt Ltd. This is an open access article under the CC BY license (http://creativecommons. org/licenses/by/4. 0/) DOI: http://dx.doi.org/10.22159/ajpcr.2018.v11i12.28855

\section{INTRODUCTION}

Periodic evaluation of drug utilization patterns needs to be done to enable appropriate modifications in the prescription of drugs which will lead to an increase in the therapeutic benefit and decrease the adverse effects. The study of prescribing patterns seeks to monitor, evaluate, provide feedback, and suggest modifications in the prescribing behavior of medical practitioners to make medical care rational and cost-effective. Hence, the evaluation of drug utilization is important for clinical, economic, and educational purposes [1]. In developing countries like India, where the financial resources are scarce and the affordability of the patients is less, implementation of the rational use of medicines becomes important [2]. International agencies such as the WHO and International Network of Rational Utilization of Drugs have recognized the enormous potential of drug utilization studies in the promotion of rational drug use and have applied themselves to evolve standard drug use indicators and data collection methods [3].

The prescribing pattern in orthopedics needs to be regularly monitored since most of the drugs prescribed carry untoward adverse effects. Earlier research conducted with similar objectives has revealed that drugs commonly prescribed in orthopedics department were nonsteroidal anti-inflammatory drugs (NSAIDs), antibiotics, and ulcer protectives [4]. Studies have shown that commonly prescribed NSAIDs in orthopedics are associated with increased risk of hospitalization and death from gastrointestinal bleeding and perforation [5]. The excessive use of these drugs may result in serious adverse events diminishing their pharmacological effect. A prescription-based survey is considered one of the most effective methods to assess and evaluate the prescribing attitude of physicians and dispensing practice [6].

Several studies of prescription patterns in the outpatient orthopedic department are available, but data on drug utilization pattern in orthopedic in-patients are scarce. Drug utilization studies conducted in the in-patient settings are effective tools that help in evaluating drug prescribing trends, efficiency, and cost-effectiveness of hospital formularies [7]. Hence, the present study was conducted with an objective to obtain information on prescribing pattern in orthopedic inpatients and to evaluate the data for the WHO core indicators regarding prescribing practices to promote rational prescribing.

The indicators enable the investigator to identify the prescription profile and quality of services offered to the population. The prescribing indicators are as follows: $[8,9]$.

1. The average number of drugs per encounter: Through this indicator, it is possible to investigate poly medication, a major factor contributing to drug interactions and adverse reactions. The educational and informational level of the person prescribing the medication may also be observed.

2. Percentage of drugs prescribed by generic name: This indicator enables the investigator to calculate the number of prescriptions in which the drugs are prescribed by the generic name. This helps in controlling drug costs in the health service. It also evaluates the influence of marketing on the person prescribing drugs.

3. Percentage of encounters with antibiotics prescribed: This indicator aims to evaluate the use of antibiotics in excess, a 
practice which contributes to bacterial dissemination and resistance.

4. Percentage of encounters with injections prescribed: This indicator helps to evaluate the injectables in excess, administration of which may have serious consequences when prescribed or applied wrongly, such as in the event of anaphylactic reactions, adverse reactions, and necrosis.

5. Percentage of drugs prescribed from the essential drugs list or formulary: This indicator helps in measuring the degree to which practices conform to the national drug policy. Besides controlling costs, the essential drugs list guarantees the treatment of the principal diseases of the population.

\section{MATERIALS AND METHODS}

\section{Study design}

A retrospective, observational study was carried out in the orthopedic department, in a tertiary care teaching hospital, Mangalore, after obtaining the permission from the Institutional Ethics Committee. The patient case files admitted in orthopedic unit during the period from July to December 2015 were included in the study. The data was collected over a period of 3 months.

\section{Collection of data}

The orthopedic in-patient case files were retrieved from the medical records department of a tertiary care teaching hospital, Mangalore. The data were documented in a predesigned case record form. It comprised patient demographics, clinical diagnosis, duration of hospital stay, and details on drugs prescribed which included total number of drugs prescribed, name and group of drugs, route of administration, number of antibiotics prescribed, number of fixed-dose combinations (FDCs), generic names of drugs (from Indian drug review 2015), and whether present in national list of essential medicines (NLEM, 2015) or not.

The data were analyzed for the WHO core prescribing indicators [9]. The formula for measurement of prescribing indicators, taken from the WHOs guide for the assessment of prescribing indicators was as follows:

1. The average number of drugs per encounter: Calculated by dividing the total number of different drug products prescribed, by the number of encounters surveyed. It is not relevant whether the patient actually received the drugs.

2. Percentage of drugs prescribed by generic name: Calculated by dividing the number of drugs prescribed by generic name by the total number of drugs prescribed, multiplied by 100 .

3. Percentage of encounters with antibiotics prescribed: Calculated by dividing the number of prescriptions in which antibiotics were prescribed by the total number of prescriptions studied, multiplied by 100 .

4. Percentage of encounters with injections prescribed: Calculated by dividing the number of prescriptions in which injections were prescribed by the total number of prescriptions studied, multiplied by 100 .

5. Percentage of drugs prescribed from essential drugs list or formulary (NLEM): Calculated by dividing the number of products prescribed which are listed on the essential drugs list or local formulary (or which are equivalent to drugs on the list) by the total number of products prescribed, multiplied by 100 .

In addition to the above indicators, prescriptions were also analyzed for the most common indication, the group of drug prescribed more commonly, the percentage of FDCs prescribed, and the most common FDCs.

\section{Statistical analysis}

The analysis was carried out by descriptive statistics using SPSS Version 20 . The results are expressed as mean \pm standard deviation, frequency, or percentages as applicable.

\section{RESULTS}

Of the 379 in-patients prescription assessed during the study period of 3 months, 221 (58.3\%) were male and 158 (41.7\%) were female. The age of the patients admitted in orthopedic unit ranged from 3 to 95 years with the majority in the age group of $40-60$ years $(38.7 \%)$, followed by $20-40$ years $(27.5 \%), 60-80$ years $(20.3 \%),<20$ years $(12.1 \%)$, and more than 80 years $(1.4 \%)$. Although patients with different ailments approached the orthopedic department, the most common indication for admission was acute trauma (57.8\%) which comprised fractures, tendon damage, and disc prolapse followed by inflammatory conditions $(26.6 \%)$ that included rheumatoid arthritis, osteoarthritis, lower back pain, and others as follows in Table 1.

The total number of drugs prescribed in the prescriptions analyzed was 2195. The average number of drugs prescribed per prescription was $5.79 \pm 2.59$. Analysis for polypharmacy showed that 164 prescriptions contained 3-5 drugs. The average duration for hospitalization was 7-11 days. The most commonly prescribed group was analgesics comprising $27.28 \%$, followed by multivitamins $(20.68 \%)$ and gastroprotectives like proton-pump inhibitors (17.12\%) as listed in Table 2. Of the analgesics, the commonly prescribed was nonsteroidal anti-inflammatory drugs (NSAIDs) (86.81\%) followed by opioids (13.19\%). Diclofenac sodium was the most commonly prescribed NSAID (230/44.23\%). Antibiotics comprised $16.12 \%$ of the drugs prescribed, the most commonly prescribed group being cephalosporin $(73.44 \%)$

Of the 2195 drugs prescribed, 885(40.31\%) were FDCs. The most common FDCs prescribed were multivitamins $(47.68 \%)$ followed by analgesics and antibiotics (Fig. 1). Majority of drugs were administered by oral route (65.80\%) followed by parenteral (33.8\%) as depicted in Fig. 2.

\section{WHO recommended prescribing indicators}

Analysis for the WHO recommended prescribing indicators as presented in Table 3 showed that the average number of drugs per prescription was $5.79 \pm 2.59$. Antibiotics were prescribed in $229(60.4 \%)$ of the prescriptions. The number of encounters with an injection prescribed was 289 (76.3\%). Use of drugs by generic name was 169 (7.7\%) and only 966 (44\%) drugs were from NLEM 2015.

\section{DISCUSSION}

The demographic details of patients showed that the majority were male. The maximum number of patients were in the age group of 4060 years and the common indication for hospitalization was fractures, tendon damage, and disc prolapse which can be manifestation of lifestyle or the physiological age-related changes that occur which is comparable to earlier studies by Srividya et al., and Wadagbalkar et al conductedin similar settings $[4,10]$.

Table 1: Common indications for orthopedic admissions

\begin{tabular}{ll}
\hline Disease & Number of prescriptions (\%) \\
\hline Acute trauma & $219(57.8)$ \\
Inflammatory conditions & $101(26.6)$ \\
Chronic trauma & $17(4.5)$ \\
Cancer & $7(1.8)$ \\
Miscellaneous & $35(9.2)$ \\
\hline
\end{tabular}

Table 2: Different categories of drugs in prescriptions analyzed

\begin{tabular}{ll}
\hline Category of drug & Number (\%) \\
\hline Analgesics & $599(27.28)$ \\
Anti-peptic ulcer agents & $376(17.12)$ \\
Antimicrobial agents & $354(16.12)$ \\
Multivitamins & $454(20.68)$ \\
Drugs for neurological disorders & $99(4.51)$ \\
Drugs for cardiovascular disorders & $90(4.10)$ \\
Drugs for endocrine abnormalities & $56(2.55)$ \\
Cold and cough remedies & $30(0.13)$ \\
Miscellaneous & $137(6.24)$ \\
\hline
\end{tabular}


Table 3: Analysis using the WHO prescribing indicators

\begin{tabular}{lll}
\hline Prescribing indicators assessed & Number (average/\%) & WHO reference value[9] \\
\hline Average number of drugs per prescription & $5.79 \pm 2.59$ & $1.6-1.8$ \\
Percentage of encounters with antibiotics prescribed & $229(60.4)$ & $20-26.8 \%$ \\
Percentage of encounters with injections prescribed & $289(76.3)$ & $13.4-24.1 \%$ \\
Percentage of drugs prescribed by generic name & $169(7.7)$ & $100 \%$ \\
Percentage of drugs prescribed from NLEM 2015 & $966(44)$ & $100 \%$ \\
\hline
\end{tabular}

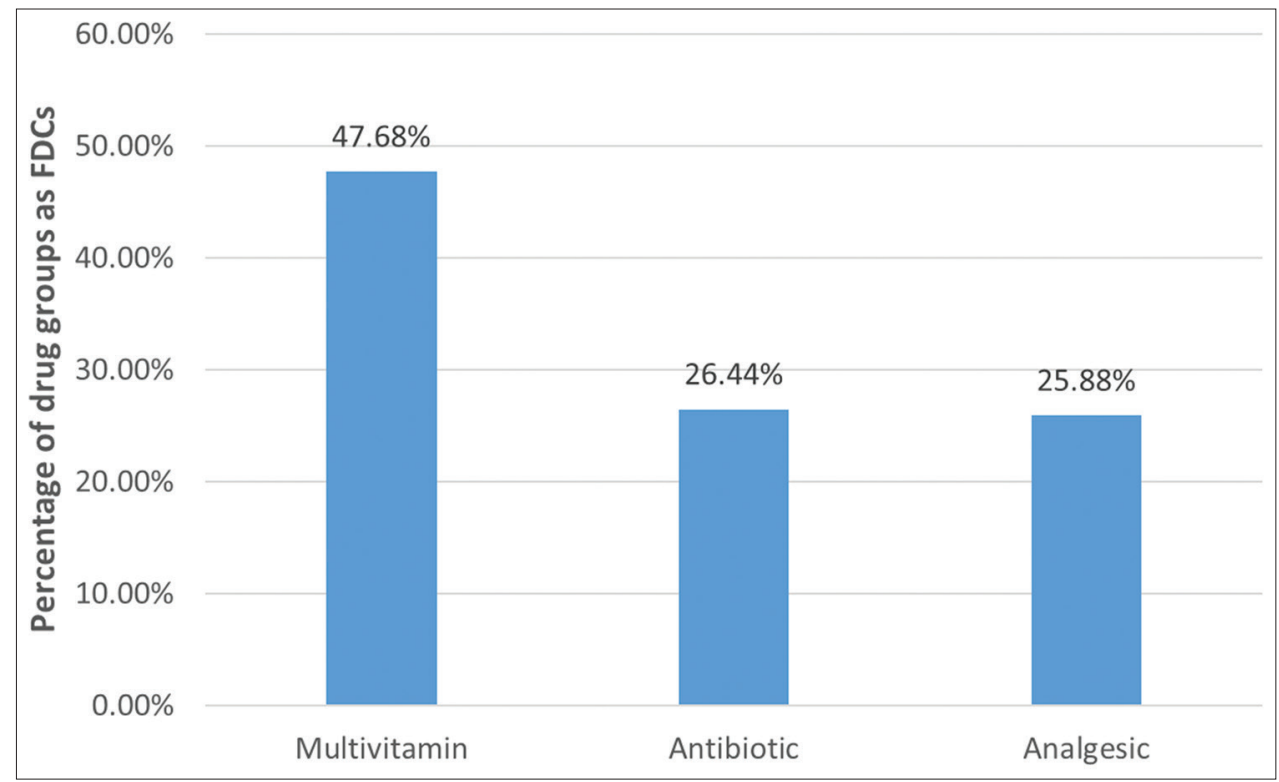

Fig. 1: The common groups of fixed-dose combinations prescribed

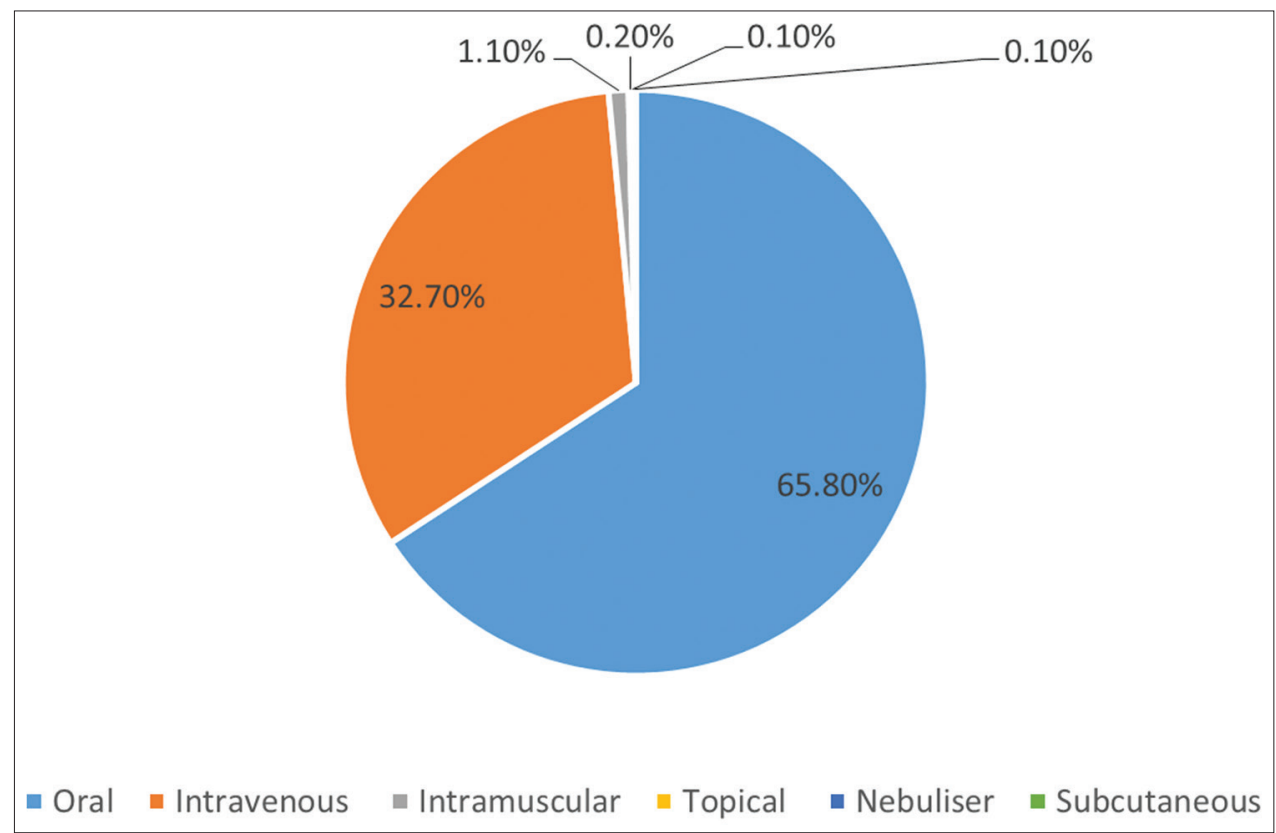

Fig. 2: Percentage distribution of various routes of administration of drugs

The study highlighted that the most commonly prescribed group of drugs was analgesics as most of the patients in orthopedic ward experience pain and inflammation. NSAIDs (86.81\%) were more common than opioids like tramadol. The result was in concordance with the earlier study by Nagla and Wadagbalkar [10]. Although NSAIDs are widely prescribed, their major limitation is gastrointestinal toxicity. Hence, in the present study, gastroprotective agents were prescribed in most of the in-patients to reduce or prevent the gastrointestinal irritation caused by NSAIDs. We noted that $17.12 \%$ of the drugs were gastroprotective agents like proton-pump inhibitors and the same has been reported from an earlier study [7]. Multivitamins with calcium constituted $20.68 \%$ of the total drugs prescribed which may be because most of the patients suffered from bone-related disorder. 230 prescriptions contained at least one or more antibiotics, as bone-related infections and bone and 
soft tissue infections following surgery are highly prevalent and serious problems encountered in orthopedics [11]. Antibiotics are prescribed as pre-operative prophylaxis and also to treat current infections. The most frequently prescribed antimicrobial agents belong to the group cephalosporin which is in alignment to study conducted by Ghosh $e t$ al. describing antibiotic usage in orthopedic department [12].

The WHO core prescribing indicators measure the performance of health-care providers in various dimensions related to the appropriate use of drugs. Based on the WHO prescribing indicators, most of the prescriptions encountered in this study were deficient in the standard requirement set by the $\mathrm{WHO}$, similar findings were reported by studies done earlier $[2,10,13]$. The WHO suggested prescribing indicators consider an average number of drugs per prescription as an important parameter indicating polypharmacy. There is no general agreement on the definition of polypharmacy, most studies consider polypharmacy as the use of multiple drugs by a single person [14]. The number of drugs per prescriptions should be kept as low as possible, particularly in orthopedic setting where higher figures culminate in increased risk of adverse drug reactions, errors of prescribing, drug interactions, decrease in efficacy, reduce patient compliance, and increased hospital cost [14,15]. In our study, the average number of drugs per prescription was $5.79 \pm 2.59$ which is higher than the WHO recommended values of 1.6-1.8 but in concordance with earlier studies including orthopedic in-patients $[2,4,10,16]$. Hence, the result from our study reflects polypharmacy, but this can be attributed to the in-patient prescription included and as the majority of patients in this study were in the age group of 40-60 years, drugs prescribed for their comorbid conditions might have increased the number of drugs per encounter. However, it indicates that educational intervention in principles of rational drug treatment needs to be introduced.

The percentage of encounters with antibiotics was $60.4 \%$ which was more than the previous study by Srividya et al. [4] and the WHO standard value of $20.0-26.8 \%$ which can be associated to the infections following soft tissues injuries and operative procedures on bone-related ailments. It should be noted that under and overprescribing can pose significant problems in clinical practice. More emphasis should be laid on antibiotic prescribing that may help reduce the development of drug resistance in the community. Minimum use of injectables should be advised because this can reduce the risk of infection through parenteral route and cost of the health care as they are associated with problems of administration and medications errors. In the present study, the number of prescriptions with injectables $(76.3 \%)$ was higher than the WHO standard of 13.4-24.1\%. The percentage of encounters with injections was higher than other studies reported in the literature $[4,10]$. The use of injections might be minimal in an outpatient department, but since it is an in-patient prescription study wherein patients are diagnosed with an acute illness in need of immediate management, the high percentage of injectable use can be attributed to the same.

In spite of various benefits such as the low cost of drug therapy, increased patient adherence, and equivalent therapeutic benefits as brand-name alternatives, generic prescribing is not a common practice in India [17]. The WHO recommends that all drugs (100\%) written on prescription should be in generic name, whereas, in our study, it was found to be $7.7 \%$ which is very low. Other studies also confirm the low percentage of generic drug prescribing $[10,18]$. There is a need to encourage generic prescribing as it works out to be economical for the patient and the possibility of drug errors is reduced. Prescribing by generic name promotes the rational use of drugs with regard to safety, efficacy, and cost.

Essential medicines list has been shown to improve the quality and cost-effectiveness of health-care delivery when combined with proper procurement policies and good prescribing practices. It measures the degree to which practices conform to national drug policy. Of the 2195 drugs, only 44\% were prescribed from the NLEM 2015 which is lower than the value specified $(100 \%)$ by the WHO. Similar results were obtained from other studies $[10,16]$. There is a need to focus on the essential drug list and how they influence the health-care system. Health-care providers should be aware of this and use of drugs from the essential drug list should be promoted for optimal use of limited financial resources, to have acceptable safety and to satisfy the health needs of the majority of the population.

About $40.31 \%$ of drugs which comprised of multivitamins, analgesics, and antibiotics were prescribed as FDCs. This is higher than noted in previous studies $[4,10]$. Irrational, inappropriate use of unwanted drugs in the combination may lead to adverse effect or drug interactions. Sensible use of FDCs should be avoided unless necessary [19]. Education and awareness to improve prescribing practices at different health-care levels may be deemed necessary.

\section{CONCLUSION}

The study provides an insight into the pattern of drug use in orthopedic in-patient of a tertiary care teaching hospital. The percentage of drugs prescribed in generic name and from essential drug lists is low compared to the WHO standards. It highlights the importance of emphasizing rational drug prescribing and toward improving awareness of the physicians and medical students to the WHO recommended standards on prescribing indicators. The information from the study would help both the prescriber and institutional authorities to review their prescribing practices and modify if necessary to facilitate better healthcare delivery.

\section{AUTHORS' CONTRIBUTION}

All authors have contributed equally in conception or design of the work, data collection, data analysis and interpretation, and drafting the manuscript.

\section{CONFLICTS OF INTEREST}

The authors have no conflicts of interest relevant to this article to be declared.

\section{REFERENCES}

1. Shankar PR, Pai R, Dubey AK, Upadhyay DK. Prescribing patterns in the orthopaedics outpatient department in a teaching hospital in Pokhara, Western Nepal. Kathmandu Univ Med J 2007;5:16-21.

2. Rajarathna K, Vishwanath M, Ramaswamy A, Kamath SD, Seshu S, Hosthota A, et al. Evaluation of WHO prescribing indicators among orthopaedic inpatients at a tertiary care hospital. J Chem Pharm Res 2014;6:278-80.

3. Biswas NR, Jindal S, Siddiquei MM, Maini R. Patterns of prescription and drug use in ophthalmology in a tertiary hospital in Delhi. Br J Clin Pharmacol 2001;51:267-9.

4. Srividya BP, Shashikumar NS, Amardeep G. Retrospective audit of prescription of drugs among inpatients of orthopaedic wards at medical college teaching hospital, Mandya. Natl J Physiol Pharm Pharmacol 2016;6:1-4.

5. Smalley WE, Ray WA, Daugherty JR, Griffin MR. Nonsteroidal antiinflammatory drugs and the incidence of hospitalization for peptic ulcer disease in elderly persons. Am J Epidemiol 1995;141:539-45.

6. Ingle PK, Patil PH, Lathi V. Study of rational prescribing and dispensing of prescriptions with non-steroidal anti-inflammatory drugs in orthopedic outpatient department. Asian J Pharm Clin Res 2015;8:278-81.

7. Choudhury DK, Bezbaruah BK. Prescribing pattern of analgesics in orthopedic in-patient department at tertiary care hospital in Guwahati, Assam, Northeast, India. Indian J Pharmacol 2016;48:377-81.

8. Vooss AT, Diefenthaeler HS. Evaluation of prescription indicators established by the WHO in Getulio Vargas-RS. Braz J Pharm Sci 2011;47:385-90.

9. Bilal AI, Osman ED, Mulugeta A. Assessment of medicines use pattern using world health organization's prescribing, patient care and health facility indicators in selected health facilities in Eastern Ethiopia. BMC Health Serv Res 2016;16:144-51.

10. Nagla A, Wadagbalkar P. Prescription pattern study of drugs given to patients admitted in orthopedic wards of a rural medical college, a 
tertiary care hospital. Eur J Pharm Med Res 2014;1:267-71.

11. Kala P, Jamuna RR, Sangeeta R. Prescription pattern of prophylactic antimicrobial agents used in preoperative patients at a tertiary care teaching hospital. Int J Pharm Pharm Sci 2018;10:128-31.

12. Kumar GA, Kumar DC, Kumar A, Ray M. Drug utilization study on antibiotics use in an orthopaedics department of a tertiary care hospital in West Bengal. J Drug Deliv Ther 2013;3:98-103.

13. Baghel R, Adwal SK, Singh V, Chourishi A. Prescribing pattern and drug utilization study in inpatients of department of orthopaedics in a rural teaching hospital of Ujjain, Madhya Pradesh, India. Int J Basic Clin Pharmacol 2018;7:1763-9.

14. Chowta MN, Adhikari PM, Raj S, Laxman M, Kariappa A, George J, et al. Evaluation of appropriateness of prescription and polypharmacy in the geriatric population: A cross-sectional study at a comprehensive geriatric clinic in a tertiary care hospital. Int J Pharm Pharm Sci 2016;8:119-23.
15. Banerjee I, Bhadury T, Agarwal M. Prescribing pattern in orthopedics outpatient department of a medical college in India. Int J Basic Clin Pharmacol 2015;4:1175-7.

16. Srihari A, Shanmukananda P, Purushotham K. Prescribing trends in the department of orthopedics of a tertiary care teaching hospital. Int J Res Pharmacol Pharmacother 2017;6:297-301.

17. Mittal N, Mittal R, Singh I, Shafiq N, Malhotra S. Drug utilisation study in a tertiary care center: Recommendations for improving hospital drug dispensing policies. Indian J Pharm Sci 2014;76:308-14.

18. Ubedulla S, Sekhar NC, Jayasree T, Shankar J, Rohit K. Prescription trends in department of orthopedics at tertiary care teaching hospital. J Chem Pharm Res 2013;5:512-7.

19. Kanagasanthosh K, Topno I. A study on recent trends of prescribing pattern in orthopaedic out-patient department from a tertiary care teaching hospital. Int J Pharm Technol 2015;6:7549-59. 